\title{
JOVENS PRATICANTES DE ATLETISMO: CONTRIBUIÇÃO DA MATURAÇÃO E VARIÁVEIS ANTROPOMÉTRICAS NO DESENVOLVIMENTO DA FORÇA EXPLOSIVA E VELOCIDADE EM PÚBERES E PÓS-PÚBERES DURANTE CINCO MESES DE TREINAMENTO
}

\author{
Thiago Mariano, Faculdade de Educação Física, Universidade Estadual de Campinas - UNICAMP, \\ Campinas, São Paulo, Brasil \\ Miguel de Arruda, Faculdade de Educação Física, Universidade Estadual de Campinas - UNICAMP, \\ Campinas, São Paulo, Brasil \\ Yuri, Muniz, Faculdade de Educação Física, Universidade Estadual de Campinas - UNICAMP, \\ Campinas, São Paulo, Brasil \\ Eduardo Henrique Pascoal Frazilli, Faculdade de Educação Física, Universidade Estadual de \\ Campinas - UNICAMP, Campinas, São Paulo, Brasil \\ Evandro Lazari, Faculdade de Educação Física, Universidade Estadual de Campinas - UNICAMP, \\ Campinas, São Paulo, Brasil
}

\section{RESUMO}

O objetivo do estudo é estimar a contribuição dos estatutos maturacionais, na variação do percentual de gordura, peso e estatura, como também na variação dos desempenhos de força explosiva, força explosiva elástica e força explosiva elástica reflexa e velocidade em jovens atletas de atletismo de ambos os sexos, no período de cinco meses de treinamento. Os atletas foram divididos segundo o sexo e estagio maturacional. Cada jogador foi submetido a uma bateria de testes motores, bem como a avaliação da composição corporal e auto-avaliação de maturação pelo teste de Tanner. Concluiu-se que ocorrem alterações distintas dependendo da variável estudada, gênero e estagio de maturação do atleta. As variáveis antropométricas variaram de acordo com o que ocorre no processo de maturação normal de indivíduos na fase púbere e pós-púberes.

Palavras-Chave: Força explosiva, Maturação, Atletismo. 


\title{
ATHLETICS YOUNG ATHLETES: CONTRIBUTION OF ANTHROPOMETRIC VARIABLES IN MATURATION AND DEVELOPMENT OF EXPLOSIVE POWER AND SPEED IN PUBERTAL AND POSTPUBERTAL DURING FIVE MONTHS OF TRAINING
}

\begin{abstract}
The objective is to estimate the contribution of maturational status, change in fat percentage, weight and height, but also the variation of the performance of explosive power, explosive strength elastic and reflex elastic explosive strength and speed in young athletes of track and field both sexes, within five months of training. The athletes were divided according to sex and maturational stage. Each player underwent a battery of motor tests, as well as assessment of body composition and self-assessment of maturity by the Tanner test. It was concluded that changes occur depending on the different variables studied, sex and stage of maturity of the athlete. Anthropometric variables varied according to what happens in the maturation process of normal individuals at puberty and post-pubescent.
\end{abstract}

KEY-WORDS: Explosive strength; Maturation; Track and field. 


\section{INTRODUÇÃO}

Diante das mudanças no desempenho físico que ocorrem ao longo do tempo na formação dos jovens atletas, tanto de ordem estrutural quanto de ordem funcional, ${ }^{1}$ e da necessidade do desenvolvimento para o alto nível da modalidade, o treinamento do atletismo tem passado cada vez mais por estudos e sistematizações de elementos relativos ao atleta e à performance.

Quanto ao atleta, a importância está relacionada principalmente ao desenvolvimento das capacidades condicionantes associadas à maturação e crescimento, ${ }^{1}$ que permitem aumentos nos saltos verticais, ${ }^{2,3}$ assim como aumentos da velocidade nas ações de deslocamento. ${ }^{4}$

As alterações significativas no rendimento do desempenho físico do salto vertical e da velocidade de deslocamentos são caracterizadas pelas mudanças na função neuro-muscular, tais como: força máxima, força explosiva, força explosiva elástica e reflexa. ${ }^{5}$

Em vários estudos foram encontrados fortes relacionamentos do desempenho do salto vertical com as manifestações de força. ${ }^{6-7}$ Neste ponto, o relacionamento entre as manifestações de força e o desempenho da velocidade de deslocamentos foram significantes e fortes ${ }^{4,6}$ apresentando valores de correlação negativa. Convém indicar que as manifestações da força vêm cada vez mais se destacando como uma variável importante no desempenho físico dos atletas. Logo, as manifestações são elementos intervenientes no desempenho físico, ou seja, treinando as capacidades condicionantes das manifestações da força geram-se aperfeiçoamentos no desempenho da velocidade de deslocamentos e no salto vertical. ${ }^{8}$

Por esse objetivo do treinamento esportivo, um fator preocupante aos estudiosos está na busca do treinamento compatível de força ao desenvolvimento do jovem atleta, que tendem a Conexões: revista da Faculdade de Educação Física da UNICAMP, Campinas, v. 9, n. 1, p. 92-108, jan./abr. 2011. ISSN: 1983-9030 
desenvolver informações sobre programa de treinamentos efetivos para a capacidade condicionante da força. ${ }^{9-16}$

Nesse contexto, o desenvolvimento da força explosiva sofre várias mudanças quanto à idade e maturação. ${ }^{1,17-19}$ Evidencias relatam a existência de aumentos lineares no desenvolvimento da força até a puberdade, ${ }^{20}$ por conseguinte, quando o jovem entra nessa fase de mudanças no desempenho da força, há a partir daí uma marcada aceleração no seu desenvolvimento. ${ }^{1,15}$

Estudos como o de Gabett et al. $^{21}$ têm demonstrado que ainda existe uma limitação de evidencias na literatura especializada, a qual visa contribuir com informações aos treinadores; isso de modo a esses componentes serem sensíveis de mudanças durante o processo de desenvolvimento e maturação. Contrariamente, outros têm sugerido que conhecendo as especificidades e sensibilidades das mudanças nos desenvolvimentos dos componentes, estes condicionam aumentos das capacidades de produção de força em jovens atletas para que possam responder de forma efetivas ao aumento do desempenho. ${ }^{1,16-17}$

Entretanto, são poucos os estudos que têm investigado a relação da maturidade sexual com a produção de força. Assim, para que seja potencializado o desempenho físico dos jovens, têm sido buscadas informações sobre as mudanças que possibilitam uma compreensão ao leitor de quais os componentes são possíveis de treinabilidade na produção da força explosiva entre as categorias e os estágios de maturidade sexual nessa fase da puberdade. Também possam responder como são as estimativas das contribuições dos fatores qualitativos e quantitativos na variação do desempenho das manifestações da força. 
No entanto, existe certa carência de estudo na literatura especializada em treinamento sobre o desenvolvimento da força (Fmax, FE, FEE, e FEER) em jovens atletas. Mediante esse fato, percebe-se analisando alguns estudos que existem mudanças diferenciadas entre jovens atletas e não atletas. Por conseguinte, nota-se que o treinamento específico de força, o desenvolvimento da força e a idade biológica têm efeitos nas mudanças do desempenho físico em pré-adolescentes, adolescentes e pós-adolescentes. ${ }^{9-10,13,22-23}$

\section{OBJETIVOS}

Estimar a contribuição dos estatutos maturacionais, na variação do percentual de gordura, peso e estatura, como também na variação dos desempenhos de força explosiva, força explosiva elástica e força explosiva elástica reflexa e velocidade em jovens atletas de atletismo de ambos os sexos, na faixa etária de 12 a 18 anos durante dois períodos distintos (período de preparação e período de competição).

\section{MATERIAIS E METODOS}

Para a amostra foram selecionados 20 atletas praticantes de atletismo de ambos os sexos de um clube de atletismo localizado na cidade de Campinas - SP. Os atletas foram divididos segundo o sexo e estagio maturacional. Cada jogador foi submetido a uma bateria de testes motores, bem como da avaliação da composição corporal por meio da coleta de medidas como peso, estatura e de dobras cutâneas subescapular, supra-ilíaca, triceptal, abdominal e panturrilha medial, segundo os procedimentos descritos por Petroski. O cálculo do percentual de gordura foi realizado segundo Boileau et al. ${ }^{24}$

As variáveis de desempenho motor foram constituídas por testes de força e velocidade. Para a capacidade de força foram coletados resultados de testes de força explosiva (FE), força Conexões: revista da Faculdade de Educação Física da UNICAMP, Campinas, v. 9, n. 1, p. 92-108, jan./abr. 2011. ISSN: 1983-9030 
explosiva elástica (FEE), força explosiva elástica reflexa (FEER). Segundo os procedimentos descritos por Bosco, ${ }^{25}$ para a FE foi utilizado o teste de salto vertical partindo de uma posição de meio agachamento em que o atleta, ao sinal do avaliador, sem auxílio dos membros superiores, salta utilizando-se somente dos componentes contráteis dos músculos, sendo este salto chamado de Squat Jump. A FEE foi analisada por meio do Countermovement jump $(C M J)$, em que o atleta, partia de uma posição em pé, e ao sinal do avaliador, fazia um movimento de meio agachamento seguido de um salto sem o auxílio dos membros superiores, que deveriam ser mantidos na cintura. Este teste visa analisar, além da capacidade contrátil da musculatura, a capacidade elástica dos músculos.

A FEER foi mensurada por meio do teste de salto vertical contínuo durante 5 segundos. Este teste foi realizado de acordo com os procedimentos descritos por Bosco e colaboradores, ${ }^{5} \mathrm{em}$ que os atletas, sem o auxílio dos membros superiores, deveriam saltar continuamente durante 5 segundos sem utilizar as articulações dos joelhos. Este teste visa analisar a capacidade dos músculos utilizados no movimento em realizar força de forma reflexa, junto com as capacidades contráteis e elásticas.

Para o teste de velocidade foram utilizadas células fotoelétricas no início e no final do percurso de uma distância de 30 metros, sendo medido o tempo gasto para que o atleta percorresse o percurso. A velocidade foi medida por células foto-elétricas. Foram executadas 3 tentativas, com intervalos de 2 minutos entre cada tentativa. A velocidade será o menor tempo realizado entre as 3 tentativas. Antes das avaliações foi realizado um aquecimento de 10 a 15 minutos. 
A variável da maturação biológica foi observada através da avaliação do estágio de maturidade sexual, a qual foi determinada pelas medidas do desenvolvimento de caracteres sexuais secundários, seguindo o procedimento descrito por Tanner. ${ }^{26}$ Os estágios de maturidade sexual serão pré-púberes (PR), púberes (PU) e pós-púberes (PO).

Foram realizadas duas coletas de dados, a primeira no mês de Agosto de 2009 e outra no mês de Dezembro. Os critérios de exclusão dos atletas para a realização dos testes foram a participação de todos os testes nas duas coletas de dados, e autorização da comissão técnica e da equipe médica do clube para a realização dos testes.

Para análise dos dados, foi utilizado o Teste-t de Students para amostras dependentes, a fim de verificar as mudanças ocorridas no peso, estatura, $\% \mathrm{G}$ e testes motores durante o semestre, a fim de verificar a magnitude de variação. Para o tratamento estatístico dos dados obtidos foi utilizado pelo software BioEstat 5.0.

\section{RESULTADOS E DICUSSÕES}

Abaixo seguem as tabelas da estatística descritiva geral da antropometria e testes físicos realizados até o momento:

Tabela 1 - Estatística descritiva da equipe feminina PU

\begin{tabular}{lrrrrrrrr}
\hline & \multicolumn{4}{c}{ Primeira Coleta } & \multicolumn{5}{c}{ Segunda Coleta } \\
\hline & Média & DP & Min. & Max. & Média & DP & Min. & Max. \\
\hline Estatura & 160.16 & 8.38 & 148.00 & 171.40 & 161.70 & 7.25 & 151.20 & 171.30 \\
Peso & 47.62 & 11.00 & 32.60 & 62.00 & 49.06 & 10.68 & 34.20 & 63.00 \\
\%G & 15.60 & 3.43 & 11.00 & 19.00 & 15.60 & 3.64 & 12.00 & 20.00 \\
SJ & 25.40 & 4.15 & 21.00 & 32.00 & 25.80 & 4.26 & 22.00 & 33.00 \\
CMJ & 26.20 & 3.83 & 22.00 & 31.00 & 27.20 & 2.77 & 25.00 & 32.00 \\
CJ5s & 22.80 & 4.20 & 17.00 & 27.00 & 25.80 & 5.21 & 18.00 & 32.00 \\
30m & 4.60 & 0.54 & 4.00 & 5.00 & 4.60 & 0.5 & 4.00 & 5.00 \\
\hline
\end{tabular}


Tabela 2 - Estatística descritiva da equipe feminina PO

\begin{tabular}{lrrrrrrrr}
\hline & \multicolumn{3}{c}{ Primeira Coleta } & \multicolumn{4}{c}{ Segunda Coleta } \\
\hline & Média & DP & Min. & Max. & Média & DP & Min. & Max. \\
\hline Estatura & 162.20 & 8.61 & 154.50 & 176.50 & 163.08 & 7.96 & 157.00 & 176.50 \\
Peso & 51.04 & 9.97 & 39.80 & 67.00 & 52.00 & 9.30 & 42.00 & 67.00 \\
\%G & 15.40 & 6.18 & 9.00 & 23.00 & 18.00 & 5.24 & 14.00 & 27.00 \\
SJ & 26.40 & 1.67 & 24.00 & 28.00 & 27.20 & 2.49 & 23.00 & 29.0 \\
CMJ & 31.00 & 3.24 & 28.00 & 35.00 & 31.60 & 3.84 & 27.00 & 36.00 \\
CJ5s & 27.20 & 2.77 & 24.00 & 30.00 & 28.60 & 2.51 & 26.00 & 31.00 \\
30m & 4.40 & 0.54 & 4.00 & 5.00 & 4.37 & 0.54 & 4.00 & 4.56 \\
\hline
\end{tabular}

Tabela 3 - Estatística descritiva da equipe masculina PU

\begin{tabular}{lrrrrrrrr}
\hline \multicolumn{3}{c}{ Primeira Coleta } & \multicolumn{5}{c}{ Segunda Coleta } \\
\hline & Média & DP & Min. & Max. & Média & DP & Min. & Max. \\
\hline Estatura & 169.33 & 10.76 & 148.00 & 177.00 & 170.00 & 9.59 & 151.00 & 177.00 \\
Peso & 57.33 & 10.82 & 37.00 & 69.00 & 56.33 & 10.23 & 37.00 & 66.00 \\
\%G & 13.67 & 5.39 & 9.00 & 22.00 & 13.17 & 6.24 & 8.00 & 25.00 \\
SJ & 29.33 & 5.68 & 21.00 & 36.00 & 30.67 & 7.39 & 21.00 & 43.00 \\
CMJ & 34.00 & 6.48 & 27.00 & 42.00 & 34.67 & 6.74 & 28.00 & 45.00 \\
CJ5s & 29.17 & 5.85 & 23.00 & 38.00 & 31.67 & 6.41 & 24.00 & 41.00 \\
30m & 4.68 & 3.02 & 4.02 & 5.02 & 4.60 & 3.50 & 4.0 & 4.67 \\
\hline
\end{tabular}

Tabela 4.0 - Estatística descritiva da equipe masculina PO

\begin{tabular}{lrrrrrrrr}
\hline & \multicolumn{3}{c}{ Primeira Coleta } & \multicolumn{4}{c}{ Segunda Coleta } \\
\hline & Média & DP & Min. & Max. & Média & DP & Min. & Max. \\
\hline Estatura & 171.75 & 11.03 & 164.00 & 179.00 & 172.75 & 7.23 & 165.00 & 179.00 \\
Peso & 60.25 & 7.04 & 51.00 & 68.00 & 57.00 & 4.24 & 52.00 & 61.00 \\
\%G & 13.05 & 1.83 & 9.00 & 22.00 & 12.75 & 7.14 & 7.00 & 23.00 \\
SJ & 29.25 & 2.99 & 26.00 & 33.00 & 30.75 & 3.95 & 26.00 & 34.00 \\
CMJ & 33.00 & 4.32 & 29.00 & 39.00 & 35.00 & 5.35 & 29.00 & 40.00 \\
CJ5s & 27.75 & 6.24 & 21.00 & 36.00 & 31.75 & 8.26 & 22.00 & 40.00 \\
30m & 4.52 & 1.52 & 4.20 & 4.60 & 4.48 & 1.89 & 4.30 & 4.6 \\
\hline
\end{tabular}

A seguir são apresentadas as tabelas do Teste-t de Students para amostras dependentes de todos os atletas separando as tabelas por gênero e estágio de maturação, sendo o valor dentro das tabelas o valor de t. 
Tabela 5 - Teste $\mathrm{t}$ das variáveis estudadas do grupo feminino (PU)

\begin{tabular}{rrrrrrrr} 
& Estatura & Peso & $\% \mathrm{G}$ & $\mathrm{SJ}$ & $\mathrm{CMJ}$ & $\mathrm{CJ} 5 \mathrm{~s}$ & $30 \mathrm{~m}$ \\
\hline $\mathrm{t}$ ( ) & $-2.79 *$ & $-6.97 *$ & 0.04 & -1.00 & -1.05 & $-3.16^{*}$ & 1.10 \\
\hline
\end{tabular}

Legenda: ${ }^{*} p \leq 0,05$

Tabela 6 - Teste t das variáveis estudadas do grupo feminino (PO)

\begin{tabular}{clllllll} 
& Estatura & Peso & $\% G$ & SJ & CMJ & CJ5s & $30 m$ \\
\hline (t) & -2.07 & -2.56 & -1.62 & -1.63 & -0.73 & -1.42 & 1.21 \\
\hline
\end{tabular}

Tabela 7 - Teste t das variáveis estudadas do grupo masculino (PU)

\begin{tabular}{rrrrrrrr} 
& Estatura & Peso & $\% \mathrm{G}$ & $\mathrm{SJ}$ & $\mathrm{CMJ}$ & $\mathrm{CJ} 5 \mathrm{~s}$ & $30 \mathrm{~m}$ \\
\hline (t) & $-1.34^{*}$ & $1.22^{*}$ & 0.81 & $-1.10^{*}$ & -0.56 & $-2.71^{*}$ & 1.20 \\
\hline
\end{tabular}

\section{Legenda: ${ }^{*} p \leq 0,05$}

Tabela 8 - Teste t das variáveis estudadas do grupo masculino (PO)

\begin{tabular}{rrrrrrrr} 
& Estatura & Peso & $\% \mathrm{G}$ & $\mathrm{SJ}$ & $\mathrm{CMJ}$ & $\mathrm{CJ} 5 \mathrm{~s}$ & $30 \mathrm{~m}$ \\
\hline (t) & 0.74 & 0.97 & -0.63 & -1.73 & -1.18 & -1.19 & 1.19 \\
\hline
\end{tabular}

De acordo com a apresentação dos dados pôde-se observar, do ponto de vista das características antropométricas que houve comportamentos distintos quando distinguimos gênero e estágio de maturação dos atletas. A única variável que se manifestou de maneira igual na média de todos os grupos foi a Estatura, que apresentou um aumento em todas as médias, explicado pela idade em que os atletas se encontram, porém as alterações significativas ocorreram nos atletas que estavam na fase púbere em ambos os sexos. O fato é evidenciado pelo processo de maturação que atinge o pico na fase púbere, fazendo com que a resposta se mostre pelo estirão de crescimento.

A variável Peso ocorreu de maneira distinta no que se diz respeito às outras variáveis. Ocorreu uma distinção entre gêneros, o que ocorreu também na variável percentual de Conexões: revista da Faculdade de Educação Física da UNICAMP, Campinas, v. 9, n. 1, p. 92-108, jan./abr. 2011. ISSN: 1983-9030 
gordura. As atletas do sexo feminino apresentaram um aumento no peso e no percentual de gordura, enquanto os atletas do sexo masculino em ambos os estágios de maturação apresentaram uma queda do peso e do percentual de gordura. O que ocorre é que as meninas tendem a acumular gordura quando comparados a meninos, que desenvolvem mais massa magra, quando ambos estão na fase púbere. ${ }^{27}$ Para Malina e Bouchard, ${ }^{27}$ os meninos púberes apresentam uma diminuição de gordura subcutânea, o que contribui mais para a instabilidade da distribuição de gordura no período da puberdade. A evidência de que a maturação é o principal fator de que esse aumento de gordura nas atletas e diminuição nos atletas, é que as alterações significantes ocorreram nos atletas púberes, sendo não significantes nos pós-púberes.

Para os testes motores houve melhora em todos os testes realizados (SJ, CMJ, CJ5s e 30m). Para tanto essa variação pode ser explicada de duas maneiras, pelo treinamento físico ou pelo estágio maturacional, e no caso do presente estudo a causa dessa variação correu pela junção das duas causas. Portanto não sendo possível uma explicação exata do quanto a maturação auxiliou no aumento do desempenho nos teste e quanto a força auxiliou nessa melhora. O aumento dos desempenhos nos testes motores ocorreu, porém somente o teste CJ5s, que condiz com a medição da força explosiva elástica reflexa, alterou de forma significantes na fase púbere em ambos os sexos. E o teste SJ que condiz com a força explosiva, alterou significantemente apenas nos púberes do sexo masculino. Essas variações encontradas nos testes de salto podem ser relacionadas diretamente ao fato de que o estágio maturacional. Para outros autores ${ }^{27-29}$ a apresentação de uma elevação mais acentuada em algumas variáveis de desempenho motor é relatada durante ou imediatamente após o período de maturação. Para Barbanti $^{30}$ as capacidades físicas que sofrem influência da maturação como a força são Conexões: revista da Faculdade de Educação Física da UNICAMP, Campinas, v. 9, n. 1, p. 92-108, jan./abr. 2011. ISSN: 1983-9030 
determinadas linearmente sendo um dos fatores influenciantes do aumento hormonal nos indivíduos do sexo masculino, hormônios esses como a testosterona e o hormônio de crescimento que são condicionantes no aumento de força, principalmente na fase da puberdade.

Outros estudos ${ }^{27,31-32}$ afirmam que processo de crescimento e desenvolvimento é responsável pelo ganho significativo no desempenho motor, entre eles o ganho de força muscular. Para Malina, ${ }^{1}$ o estatuto maturacional é um fator que contribui positivamente no nível de desenvolvimento da força muscular.

O aumento da força explosiva apenas nos atletas púberes do sexo masculino pode ser explicado pelo fato de que os meninos aumentam sua massa muscular durante a puberdade, fato que aumenta a produção de força nesse período da vida do atleta.

Com base nesses estudos aqui indicados, nota-se que a variação nos desempenhos de força entre as distintas categorias analisadas é influenciada diretamente pelo estágio de crescimento e desenvolvimento em que o jovem atleta se encontra como também pelo treinamento em que ele foi submetido.

\section{CONCLUSÕES}

Com isso conclui-se que ocorrem alterações distintas dependendo da variável estudada, gênero e estagio de maturação do atleta. As variáveis antropométricas variaram de acordo com o que ocorre no processo de maturação normal de indivíduos na fase púbere e pós-púberes. 
No que se diz respeito aos testes motores houve melhora em todas as médias dos testes realizados. Porém somente os testes de CJ5s obteve uma alteração estatisticamente significante em púberes de ambos os sexos e o teste SJ com uma variação significante apenas em púberes masculinos.

O estudo mostrou que apenas as alterações significantes ocorreram apenas nos púberes, sendo que no pós-púberes ocorreram alterações porém não estatisticamente significante, no que se diz a algumas variáveis estudas, tais como Peso, Estatura e os testes motores de CJ5s e SJ no masculino.

Vale dizer que o projeto inicial não foi concluído pois seria examinado mais uma coleta de dados. Como também os voluntários da pesquisa diminuíram significantemente pelos critérios de exclusão imposta pela pesquisa.

Por fim gostaria de agradecer ao professor Evandro e a toda equipe técnica da ORCAMPI-UNIMED pela oportunidade de estarmos ajudando e coletando os dados da pesquisa no clube. Agradeço ao professor Miguel de Arruda pela constante orientação, ajuda e preocupação durante todo o transcorrer da pesquisa.

\section{REFERENCIAS}

${ }^{1}$ MALINA, R. M. et al. Maturity-associated variation in sport-specific skills of youth soccer players aged 13-15 years. Journal Sports Science, v.23, p. 515-22, 2005.

${ }^{2}$ SCATES, A. L.; LINN, M. Complete conditioning for volleyball. Champaign: Human Kinetics, 2003. 
${ }^{3}$ MALATESTA, D. et al. Effecs of electromyostimulation training and volleyball practice on jumping ability. Journal of Strength and Conditioning Research, v. 17, n. 3, p. 573-579, 2003.

${ }^{4}$ NUNES, C. G. Associação entre força explosiva e a velocidade de deslocamento em futebolistas profissionais. 2004. Dissertação (Mestrado) - Faculdade de Educação Física, Universidade Estadual de Campinas, Campinas, 2004.

${ }^{5} \mathrm{BOSCO}$, C. et al. Effetto della vibrazione su forza esplosiva, resisteza Allá forza veloce e flessibilità muscolare. Medicina Dello Sport, v.54, p. 287-293, 2001.

${ }^{6}$ YOUNG, W.; MCLEAN, B.; ARDAGNA, J. Relationship between strength qualities and sprinting performance. Journal of Sports Medicine and Physical Fitness, v.35, p.13-19, 1995.

${ }^{7}$ CICCARONE, G. et al. Evaluation of jumping capacities in high-level volleyball players. In: CONGRESS OF THE EUROPEAN COLLEGE OF SPORT SCIENCE, 6, 2001, Cologne. Abstract... Cologne: German Society of Sport Science, 2001.

${ }^{8}$ KRAEMER, W. J.; HÄKKINEN, K. Treinamento de força para o esporte. Porto Alegre: Artmed, 2004. 
${ }^{9}$ HÄKKINEN, K.; KESKINEN, K.L. Muscle cross sectional area and voluntary force production characteristics in elite strength and endurance trained athletes and sprinters. European Journal of Applied Physiology, v. 59, p. 215-220, 1989.

${ }^{10}$ BLIMKIE, C. J. Resistance during training pre-and early puberty: efficacy, trainability, mechanism, and persistence. Canadian Journal Sport Science, v.17, n.4, p.264-279, 1992.

${ }^{11}$ OZMUN, J. C, MIKESKY, A. E.; SURBURG, P. R. Neuromuscular adaptations following prepubescent strength training. Medicine and Science in Sports and Exercise, v.26, p.510-514, 1994.

${ }^{12}$ FAIGENBAUM, A. D.; WESCOTT, W. L.; MICHELI. L. J.; The effects of strength training and detraining on children. Journal of Strength and Conditioning Research, v.10, p.109-114, 1996.

${ }^{13}$ BLIMKIE, C. J. R.; SALE, D. G. Strength development and trainability during childhood. In: VAN PRAAGH, E. Pediatric anaerobic performance. Champaign: Human Kinetics, 1998. chapter 9. p.193-224.

${ }^{14}$ FAIGENBAUM, A. D. et al. The effects of different resistance training protocols on muscular strength and endurance development in children. Pediatrics, v. 104, n. 5, p.17, 1999. 
${ }^{15}$ MANNO, R.; GIMINIANI, R. D. Controllo e allenamento della forza muscolare nei bambini e nei giovani. AtleticaStudi, v.3/4, p.27-40, 2003.

${ }^{18}$ MARTIN, R. J. F. et al. M. Longitudinal changes of maximal short-term peak power in girls and boys during growth. Medicine and Science in Sports and Exercise, v. 36, n.3, p. 498-503, 2004.

${ }^{16}$ FAIGENBAUM, A. D.; MILIKEN, L. A.; WESTCOTT, W. L. Maximal strength testing in healthy children. J Strength Cond Res, v. 17, p.162-166, 2003.

${ }^{17}$ BERALDO, S. Il miglioramento della forza nell' adolescenza. AtleticaStudi, v. 314, p. 6574, 2003.

${ }^{19}$ ARRUDA, M.; HESPANHOL, J. E.; SILVA NETO, L. G. Força explosiva em jovens atletas do sexo masculino. Revista Perfil, v. 7, n. 8, p. 73-4, 2005.

${ }^{20}$ MALINA, R. M.; BAR-OR, O. Growth, maturation, and physical activity: Champaign:Human Kinetics, 2004.

${ }^{21}$ GABETTT, T. et al. Changes in skill and physical fitness following training in talentidentified volleyball player. Journal of Strength Conditioning Research, v. 20, n. 13, p. 29-35, 2006. 
${ }^{22}$ MERO, A.; JAKKOLA, L.; KOMI, P. V. Serum hormones and physiological performance capacity in young boys athletes during a 1 years training period, European Journal of Applied Physiology, v. 60, n. 2, p. 32-37, 1990.

${ }^{23}$ RAMSAY, J. A. et al. Strength effects in prepubescent boys. Medicine and Science in Sports and Exercise, v. 14, n. 1, p. 134-143, 1990.

${ }^{24}$ BOILEAU, R; LOHMAN, T.; SLAUGTHER, M. Exercice and body composition of children and youth. Scandinavian Journal Sports Science, n. 7, p. 17-27, 1985.

${ }^{25}$ BOSCO, C. La valoracion de la fuerza com el Teste de Bosco. Barcelona: Paidotribo, 1994.

${ }^{26}$ TANNER, J. M. Growth at adolescence, with a general consideration of the effects of hereditary and environmental factors upon growth and maturation from birth to maturity. 2. ed. Oxford: Blackwell, 1962

${ }^{27}$ MALINA, R. M.; BOUCHARD, C. Atividade física do atleta jovem: do crescimento à maturação. São Paulo: Rocca, 2002.

${ }^{28}$ MACHADO, D. R. L. Maturação esquelética e desempenho motor em crianças e adolescentes. 2004. Dissertação (Mestrado) - Universidade de São Paulo, São Paulo, 2004. 
${ }^{29}$ GARCIA, L. L.; RAMADA, B. A. Maturacion sexual e biológica. Anales Españoles de Pediatria, Barcelona, v. 38, n. 3, p. 245-249, 1993.

${ }^{30}$ BARBANTI, V. J. Desenvolvimento das capacidades físicas básicas na puberdade. Revista Paulista de Educação Física, São Paulo, v. 3, n. 5, p. 31-37, 1989.

${ }^{31}$ BALE, P. et al. Biological and performance variables in relation to age in male and female adolescent athletes. Journal of Sports Medicine and Physical Fitness, Turin, v. 32, n. 2, p. 142-148, 1992.

${ }^{32}$ FERREIRA, M. et al. Comparação da aptidão física de escolares de Itaquera e São Caetano do Sul. Revista Brasileira de Ciência e Movimento, São Caetano do Sul, v. 4, n. 2, p. 19-27, 1990. 